\title{
Open CrowdRE Challenges in Software Ecosystems
}

\author{
David Johnson $^{1}$, James Tizard ${ }^{2}$, Daniela Damian ${ }^{1}$, Kelly Blincoe ${ }^{2}$, Tony Clear ${ }^{3}$ \\ ${ }^{1}$ University of Victoria, Canada \\ ${ }^{2}$ University of Auckland, New Zealand \\ ${ }^{3}$ Auckland University Of Technology, New Zealand \\ davidjo@uvic.ca, jtiz003@aucklanduni.ac.nz,danielad@uvic.ca, k.blincoe@auckland.ac.nz, tony.clear@aut.ac.nz
}

\begin{abstract}
CrowdRE has been argued to comprise four main activities: motivating crowd members; eliciting feedback; analysing feedback and monitoring context and usage data. However, determining requirements within a software ecosystem poses demands beyond those found by a single product owner. In this paper, we describe open challenges for CrowdRE in handling the many, competing and heterogeneous sources of $\mathrm{RE}$ data in a software ecosystem. We illustrate the case of Xero ecosystem - a global accounting software company - as an ecosystem platform provider and Figured - a developer of a farming industry application and one of Xero's partner apps.
\end{abstract}

\section{INTRODUCTION}

A software ecosystem (SECO) has been defined "as a set of businesses functioning as a unit and interacting with a shared market for software and services, together with the relationships among them. These relationships are frequently underpinned by a common technological platform or market and operate through the exchange of information, resources and artifacts." [4]. Participating in a SECO benefits both the platform provider and the collaborating software producers by facilitating these producers to adapt to opportunities and create new products and markets [6]. A network of innovation created by a SECO eases the barrier of entry for software producers and facilitates the creation of new products. There are a number of requirements engineering (RE) challenges that have been previously identified for SECOs, including challenges in requirements negotiation [7], software integration [8], governance [8], and support for emergent requirements [5, 8].

We describe open challenges for SECOs in the context of CrowdRE, resulting from the large heterogeneous user base giving requirements on multiple channels, with multiple interested parties. The Platform provider and their SECO partners must balance commercial competition with the need to cooperate in eliciting requirements from distinct crowds across the ecosystem. New communication mechanisms and (big data) analytics are needed that scale with the crowd [9]. Groen et al. defined four CrowdRE activities and their associated challenges [3]. In addition to these known CrowdRE challenges, we propose new challenges specific to platform ecosystems for each of these four activities:

1) Motivating crowd members: The SECO platform provider needs to motivate feedback from several distinct crowds. This includes motivating feedback from both their own users and the users of the partner apps, who they may not have direct access to. They must also motivate feedback from the app developers, who are users of the platform itself.

2) Eliciting feedback: Both the platform provider and app partners elicit and coordinate input from across a variety of, often disparate, feedback channels.

3) Analyzing feedback: Both the platform provider and app partners analyze feedback from the various channels, and they must identify the feedback that is relevant to each SECO party.

4) Monitoring context and usage data: Both the platform provider and app partners may monitor context or usage data to understand how the apps are being used. This can introduce significant privacy and security concerns, and brings technical challenges given the different data formats across the divergent channels.

To illustrate these challenges, we report from an exploratory study of the growing ecosystem around Xerd ${ }^{1}$, a global accounting software company with an open software platform. We describe the RE processes pertaining to the platform provider as well as one of its partner apps, Figured ${ }^{2}$, a farming financial management system that integrates with Xero.

\section{Research Methodology}

We collected data on the Xero SECO through semistructured interviews with members of the Xero Executive Ecosystem Leadership team and one of the founders of the partner app, Figured. The exploratory interviews lasted one hour on average.

The interviewees from Xero included the Executive Ecosystem General Manager, Developer Experience General Manager, Ecosystem Partnership General Manager, Customer Experience General Manager, and Ecosystem Martketplace General Manager. In these interviews, initial questions included:

- how do you currently identify requirements for the Xero platform?

- what are the typical communication mechanisms with the several stakeholders around the Xero platform?

- what are the groups of stakeholders in the Xero SECO?

- do you experience any challenges in collecting and analysing requirements for the platform growth?

For the partner app Figured, the questions centered around the mechanisms through which they identify and analyze their

1 https://www.xero.com

${ }^{2}$ https://www.figured.com 
users' input from the ecosystem, as well as any challenges they experience in coordinating with the platform provider.

Thematic analysis was used to analyze the interview data [2]. To identify open challenges, we considered the four CrowdRE activities described by Groen et al. [3]. Using thematic analysis, we identified codes describing common things across the interview data and then mapped these themes to these four CrowdRE activities.

\section{Xero Ecosystem}

Xero offers an accounting software product for small businesses and has also created an open software platform that enables other software providers to access the Xero accounting data. This has created an ecosystem of business partners who develop software solutions that extend and enhance the Xero product. We describe this ecosystem by considering the three main components of SECOs described by Jansen et al. [4]: a common technology platform, a shared market for software and services, and the relationships between stakeholders.

In the Xero ecosystem, the common technology platform is their open proprietary platform offered through a public RESTful API. Utilizing the Xero platform, developers create applications that extend Xero's core functionality. These applications can be either private customizations or partner applications. Partner applications must be certified by Xero, but the app is then publicly available in the Xero App Marketplace. Currently, there are more than 750 partner apps in the Xero app marketplace. The Xero product provides core accounting functionality, but through the Xero platform a SECO is enabled that provides the applications needed to run every part of a business. The platform lowers the barrier for entry for other software providers to develop applications meeting the needs of the small business market not covered by the Xero product.

Within this market, the relationships between stakeholders ensure the health of the ecosystem. One method Xero uses to manage relationships with developers is through the partner program. Building relationships with key partners is important to fostering innovation by leveraging the wisdom of the crowd that is part of the ecosystem as well as sharing the risk of entering new markets and developing new functionality.

\section{RE IN THE Xero Ecosystem}

An ecosystem adds complexity to the RE process for a software vendor such as Xero. In addition to standard product line RE, RE now extends to the Xero platform. Developers of the partner applications are direct users of the platform, and their needs are important for the ecosystem health. Xero must also look beyond their direct customers and resources for feedback during the RE process. Evaluating the needs of the ecosystem requires an understanding of users of the partner applications as well. To this end, the RE process within the Xero ecosystem now has three major focuses: RE for the Xero product, RE for the Xero platform, and RE for the Xero ecosystem. The 'crowd' includes the Xero product end-users, app developers, and the end-users of the partner applications as shown in Figure 1
TABLE I

XERO AND FIGURED REQUIREMENT CHANNELS

\begin{tabular}{|l|l|}
\hline Feedback Channel & Description \\
\hline Xero business community forum & $\begin{array}{l}\text { Feedback on Xero's integration with partner apps and } \\
\text { support for Xero's users }\end{array}$ \\
\hline $\begin{array}{l}\text { Xero developer and user voice } \\
\text { forums }\end{array}$ & Feedback and feature requests for Xero's API \\
\hline Figured feature request forum & Feature requests for Figured's product \\
\hline $\begin{array}{l}\text { Figured support page } \\
\text { (live chat bubble) }\end{array}$ & $\begin{array}{l}\text { Figured users give direct feedback and get immediate } \\
\text { support }\end{array}$ \\
\hline $\begin{array}{l}\text { Direct communication } \\
\text { (Xero and Figured) }\end{array}$ & $\begin{array}{l}\text { Requirements passed directly between parties. } \\
\text { Communication between xero and their partners } \\
\text { through the app partner program }\end{array}$ \\
\hline
\end{tabular}

Xero’s Ecosystem

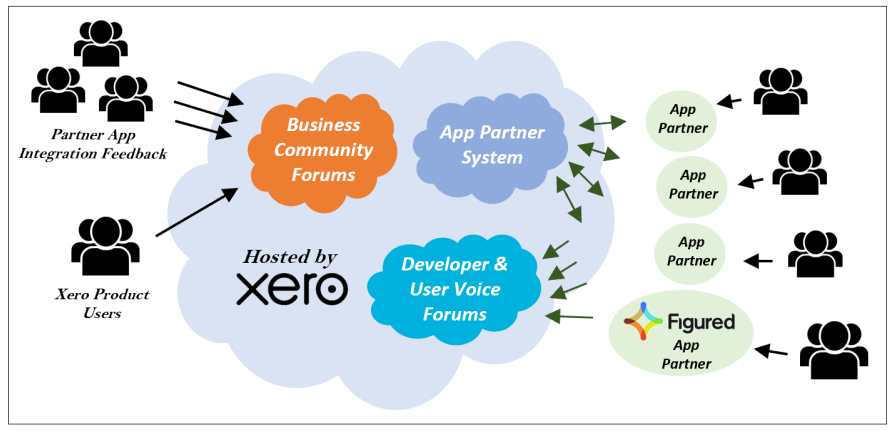

Fig. 1. Requirement flow in the Xero ecosystem

\section{A. Xero - SECO Platform Provider}

In regard to the Xero product, Xero gathers requirements through various channels including a user forum with a feature request channel and direct communication with users through sales support and conferences like Xerocon. To perform RE for the Xero platform, Xero has to communicate with app developers. Dedicated developer evangelists work directly with app developers, and they host online feedback mechanisms such as a developer forum that collects feature requests from developers. Developers also have channels for direct communication through conferences and the partner program.

$\mathrm{RE}$ is also needed for the overall ecosystem. Currently, Xero analyzes the ecosystem through the partner program by working directly with app partners and through the app marketplace. The app marketplace includes a rating system and written feedback for the integration quality of each partner app. Additionally, Xero has a connected app section within their general user forum. New and expanded channels for collecting ecosystem user feedback could benefit from CrowdRE.

\section{B. Figured - SECO App Partner}

Partner apps also have their own RE processes that may differ from the platform provider. We examine Figured to explore the RE needs of a Xero app partner. As described above, Figured offers farm management software that integrates with Xero. Figured has more traditional RE needs for their product, but must also look to Xero and the ecosystem for RE feedback on their integration with Xero. For the Figured product, requirements feedback from users comes through 
several traditional channels. Figured have their own feature request forum where registered users can suggest new features. They also have a chat feature on their website where users can get assistance and give feedback directly to Figured support personnel. Further, Figured collects requirements through direct communication with their end users.

The Xero ecosystem offers new feedback channels for Figured's integration requirements. First, Xero's Business Community forum contains sub-forums for each partner app where users can give a star rating and textual feedback on the quality of the app integration. Additionally, Xero's product strategy is an important source of information for partners to base their own product strategy and requirements. Partners like Figured may need to adapt their product and strategy based on Xero's strategy. There are several channels where Figured can identify Xero's requirements and strategy. Direct communication is available through Figured's relationship with Xero enabled through the app partner program, where more favourably ranked partners, as determined through criteria such as connection numbers and the number of app reviews, have greater privileges. Xero's platform roadmap is also open to the public, in the form of a Trello board, providing future strategy and directions for the SECO platform, which may influence Figured's product strategy. These new input channels for Figured's RE process increase the breadth of the information available to Figured and provide new opportunities and challenges for taking advantage of CrowdRE.

\section{Open Challenges}

CrowdRE for SECO RE brings new opportunities and challenges to the ecosystem platform provider and its partners. Using the four CrowdRE activities as described by Groen et al. [3], we describe some of the open challenges that result from the multiple and heterogeneous sources of requirements knowledge in software ecosystems. These challenges emerged from our interviews from Xero and Figured.

1) Motivating Crowd Members: Platform providers often need to monitor the health of the entire ecosystem, this requires that, in addition to their own users, they must motivate users of their partner apps to provide feedback. Feedback on a partner app can also be useful for the platform provider since it can provide insights such as problems with the app integration, issues with features of the core product, or ways the platform could be extended to provide needed functionality in the partner apps.

In some cases, the users of the partner apps may not be direct users of the core product and, thus, have no direct connection to the platform provider. The platform provider must rely on separate business entities (app partners) to motivate these end users to provide feedback. While the platform provider and app partners may be competitive in other aspects of operation, cooperation in motivating feedback is critical to maintain the health of the SECO.

Xero, for example, provides incentives to app partners who receive reviews within the Xero marketplace. Their partner program $^{3}$ is tiered, with additional benefits at each tier, and one of the requirements to move up the tiers is the number of reviews received.

An additional challenge is motivating feedback on the platform and the open APIs from the app developer 4 which can be quite different from motivating traditional end users. This adds a new 'crowd' that must be considered. Feedback from this crowd of developers is often delayed, since the developers need time to build new features using a newly delivered API before they can provide feedback. For a platform product team this can be hard and sometimes demoralising, as they might not know the true impact of the capability they have just delivered for months or even over a year. In addition to this delay in feedback, product teams must also carefully balance the needs of many developers in addition to their own strategic plans to choose which APIs to open. This inevitably means some developers will be disappointed as not all functionality will be made open through the platform. The platform provider needs to continue to motivate developers to provide feedback, even when they are unable to act on all of the feedback provided.

Open challenge 1: How can platform providers motivate feedback across the many heterogeneous 'crowds' of end users? What strategies can the platform provider implement to effectively motivate app partners to elicit feedback from users who may not be directly exposed to the core platform?

2) Eliciting Feedback: As discussed in Section IV] Xero and Figured both provide standard channels for eliciting feedback from the 'crowd' for their products. Further, Xero, as the SECO platform provider must also elicit feedback regarding the platform (i.e. the API and infrastructure) and the ecosystem. For this, Xero provides a developer feature request channel and the app marketplace review forums. However, users may be more prone to visit the app partner's website and feedback channels, rather than Xero's. Platform providers must rely on their partners to provide appropriate channels for eliciting feedback and to make that information available.

Further, the line between the core product and the partner apps (often integrations with the core product) can be blurred. End users may be unable to differentiate the features of the core product and those of the partner app when they are seamlessly integrated. End users are typically presented with multiple feedback channels, from more than one interested party, hosted on different websites (see Table 1). Users may find it difficult to identify the best channel for their feedback. For example, we identified many reviews in Xero's app marketplace not only addressing the integration with Xero (as desired), but reviewing the full partner app.

Open challenge 2: With the line between different products becoming blurred in a SECO, how should platform providers and their partners coordinate feedback elicitation?

3 https://developer.xero.com/partner/app-partner-program/

${ }^{4}$ https://devblog.xero.com/developers-as-your-customer-or-how-i-learnedto-stop-worrying-and-love-the-feedback-loop-19a3caa62aa3 
3) Analyzing Feedback: Analysis at this large-scale with many different feedback channels can be difficult. There are 750+ partners in Xero's ecosystem, therefore a large number of channels that could contain relevant feedback. Yet, useful information could be obtained through analysis of these channels, both by the platform provider and the partner apps in their innovation processes. However, analysis of the feedback needs to distinguish if the feedback is for the the platform, core product, or partner app. Additionally, analysis may need to consider the combination of apps being used by the feedback giver as the interaction between the apps can impact the experience of the end user. Partner apps may also integrate with competitor platform providers (e.g. Quickbooks or Intuit), so partner apps may have the added challenge of analysing feedback in the context of each platform integration. As the ecosystem grows, new challenges may arise in scaling existing CrowdRE analysis tools with the highly networked ecosystems and heterogeneous inputs.

Open challenge 3: With a large set of heterogeneous feedback channels, how can the interested parties identify and manage feedback relevant to their products?

4) Monitoring context and usage data: While considering the context and usage data of apps can help developers understand how the apps are being used, it also brings unique challenges in a platform ecosystem. Technical challenges arise as the data is likely to exist in different formats and channels. More importantly, it is not clear whether the platform provider should have access to this data of their partner apps. Discovering, accessing, and sharing usage data contributed by developers and users of partner apps raises security and privacy concerns. Users may not want their data shared. Further, when aggregating and collating data across the many different apps in the ecosystem, new information is likely to be revealed, which is a major data privacy concern [1].

Open challenge 4: Should platform providers be allowed to monitor context and usage data of their partner apps? If yes, how can user privacy be maintained?

\section{CONCLUSION}

We described open CrowdRE challenges faced within a software ecosystem, as input comes from an increasing number of heterogeneous channels. The discussion is grounded in the case of Xero, a global software company and ecosystem provider. Drawing on the landscape and challenges facing product owners and RE through the crowd [3], we highlight new challenges that arise within the SECO model. The insights we present from this exploratory study are a starting point in further development of an understanding of crowdRE challenges in software ecosystems. They need to be better understood to effectively design any applicable technologies that a CrowdRE strategy can utilise, to address the diverse challenges posed by ecosystem stakeholders and their needs, before tackling the aspects of scale.

\section{ACKNOWLEDGMENT}

This research was partially funded by the New Zealand Ministry of Business, Innovation and Employment (MBIE) through the Catalyst: Leaders Scheme of the Royal Society of New Zealand.

\section{REFERENCES}

[1] William A Babcock and William H Freivogel. The SAGE Guide to Key Issues in Mass Media Ethics and Law. SAGE Publications, 2015.

[2] Virginia Braun and Victoria Clarke. Thematic analysis. 2012.

[3] Eduard C Groen, Norbert Seyff, Raian Ali, Fabiano Dalpiaz, Joerg Doerr, Emitza Guzman, Mahmood Hosseini, Jordi Marco, Marc Oriol, Anna Perini, et al. The crowd in requirements engineering: The landscape and challenges. IEEE software, 34(2):44-52, 2017.

[4] Slinger Jansen, Anthony Finkelstein, and Sjaak Brinkkemper. A sense of community: A research agenda for software ecosystems. In Proceedings of the 31st International Conference on Software Engineering-Companion Volume, pages 187-190. IEEE, 2009.

[5] Eric Knauss, Aminah Yussuf, Kelly Blincoe, Daniela Damian, and Alessia Knauss. Continuous clarification and emergent requirements flows in open-commercial software ecosystems. Requirements Engineering, 23(1):97-117, 2018.

[6] Ramesh Lal and Tony Clear. Enhancing product and service capability through scaling agility in a global software vendor environment. In Proceedings of the 13th International Conference on Global Software Engineering, pages 59-68, 2018.

[7] Konstantinos Manikas and Klaus Marius Hansen. Software ecosystems-a systematic literature review. Journal of Systems and Software, 86(5):1294-1306, 2013.

[8] George Valença, Carina Alves, Virgínia Heimann, Slinger Jansen, and Sjaak Brinkkemper. Competition and collaboration in requirements engineering: A case study of an emerging software ecosystem. In Proceedings of the IEEE 22nd International Requirements Engineering Conference (RE), pages 384-393. IEEE, 2014.

[9] Karina Villela, Anne Hess, Matthias Koch, Rodrigo Falcao, Eduard C Groen, Jörg Dörr, Carol Naranjo Valero, and Achim Ebert. Towards ubiquitous re: A perspective on requirements engineering in the era of digital transformation. In Proceedings of the IEEE 26th International Requirements Engineering Conference (RE), pages 205216. IEEE, 2018. 